
Cahiers
de a Recherche
Four es Droits
Fondamentaux
Cahiers de la recherche sur les droits fondamentaux

18 | 2020

La vulnérabilité

\title{
Vulnérabilité et droit public
}

\section{Aurore Catherine et Samuel Etoa}

\section{OpenEdition}

\section{Journals}

Édition électronique

URL : https://journals.openedition.org/crdf/6407

DOI : $10.4000 /$ crdf.6407

ISSN : 2264-1246

Éditeur

Presses universitaires de Caen

Édition imprimée

Date de publication : 19 novembre 2020

Pagination : 21-28

ISBN : 978-2-84133-987-7

ISSN : 1634-8842

Référence électronique

Aurore Catherine et Samuel Etoa, «Vulnérabilité et droit public », Cahiers de la recherche sur les droits fondamentaux [En ligne], 18 | 2020, mis en ligne le 19 novembre 2021, consulté le 14 novembre 2022. URL : http://journals.openedition.org/crdf/6407 ; DOI : https://doi.org/10.4000/crdf.6407 


\title{
Vulnérabilité et droit public
}

\author{
Aurore CATHERINE \\ Maître de conférences en droit public à l'université de Caen Normandie \\ Centre de recherche sur les droits fondamentaux et les évolutions du droit (CRDFED, EA 2132) \\ Samuel ETOA \\ Maître de conférences en droit public à l'université de Caen Normandie \\ Centre de recherche sur les droits fondamentaux et les évolutions du droit (CRDFED, EA 2132)
}

I. La vulnérabilité interrogée par le droit public

A. La prise en compte implicite de la vulnérabilité

B. Les contours incertains de la notion de vulnérabilité

II. Le droit public interrogé par la vulnérabilité

A. La redéfinition des concepts fondateurs

B. Le rôle des garanties juridictionnelles

Cantonnée il y a peu encore à la sociologie ${ }^{1}$, la vulnérabilité gagne depuis une vingtaine d'années le vocabulaire juridique $^{2}$. La doctrine privatiste paraît en pointe en la matière. Ainsi la vulnérabilité est-elle depuis longtemps employée en droit pénal ${ }^{3}$. En droit civil, la loi no ${ }^{\circ}$ 2007-308 du 5 mars 2007 portant réforme de la protection juridique des majeurs semble avoir fait de la vulnérabilité un thème récurrent de discussion ${ }^{4}$. En 2009, la Cour de cassation publie un rapport consacré à la notion's. Plus récemment encore l'apparition d'un "consommateur vulnérable» constitue, dit-on, une nouvelle effigie du droit de la consommation ${ }^{6}$. Et l'on pourrait multiplier longuement les références et les travaux relatifs à la vulnérabilité en droit privé. À titre de comparaison, le droit public s'avère remarquablement peu disert. Le mot «vulnérabilité» ou son corollaire «vulnérable » n'apparaissent pour ainsi dire jamais dans les lexiques des ouvrages de droit constitutionnel ou de droit administratif. Plus surprenant, ces références sont également absentes des précis, traités et manuels relatifs aux droits de l'homme. Le Dictionnaire des droits de l'homme comporte bien une entrée dédiée à la «vulnérabilité» dans son index rerum, mais le renvoi

1. Sur ce point, voir C. Martin, «Penser la vulnérabilité. Les apports de Robert Castel», Les cahiers de la justice, n 4, 2019, p. 669-677

2. Voir notamment B. Eyraud, P. Vidal-Naquet, «La vulnérabilité saisie par le droit», Revue justice actualités, 2013, p. 3 sq.; F.-X. Roux-Demare, "La notion de vulnérabilité, approche juridique d'un concept polymorphe», Les cahiers de la justice, n 4 , 2019, p. 619-630.

3. Ainsi la vulnérabilité de la personne peut-elle être tout à la fois un fait justificatif ou une circonstance aggravante de l'infraction.

4. Il est intéressant de constater sur ce point que le terme «vulnérabilité» n'apparaît pas dans la loi du 5 mars 2007 , de sorte que son emploi dans le discours civiliste semble être le fruit d'une volonté doctrinale. Voir, en ce sens, J. Hauser, "Des incapables aux personnes vulnérables», Droit de la famille, no 5, 2007, étude 14; J. Hauser, «L’incapable, le protégé, le vulnérable», Revue trimestrielle de droit civil, 2009, p. 298. Plus largement sur le droit des majeurs protégés, voir Nouveau droit des majeurs protégés. Difficultés pratiques, G. Raoul-Cormeil (dir.), Paris, Dalloz (Thèmes et commentaires), 2012.

5. Cour de cassation, Les personnes vulnérables dans la jurisprudence de la Cour de cassation, rapport annuel de la Cour de cassation 20o9, Paris, La documentation française, 2009, en ligne: https://www.courdecassation.fr/publications_26/rapport_annuel_36/rapport_2009_3408.

6. S. Le Gac-Pech, «Le consommateur vulnérable: la nouvelle effigie du droit de la consommation », Revue Lamy droit civil, $\mathrm{n}^{\circ}$ 99, décembre 2012 , p. $61 s q$ 
opéré ne concerne que les notions d'esclavage, de servitude et de travail forcé7. La moisson est donc maigre dans les ouvrages académiques et porterait à croire que le droit public se désintéresserait de la vulnérabilité. Une telle analyse toutefois s'avère superficielle. D'abord parce que le droit public ne peut faire l'économie d'une réflexion sur le sujet. Contrairement au droit privé qui postule l'égalité des relations entre particuliers, le droit public repose quant à lui sur l'inégalité fondamentale des rapports entre la puissance publique et les individus, de sorte que le corpus juridique qui s'applique à ces rapports s'avère exorbitant du droit commun ${ }^{8}$. Une telle observation conduit à envisager le droit public comme un droit intrinsèquement fondé sur la vulnérabilité des sujets qu'il est appelé à régir; ce qui ne veut pas dire pour autant que le déséquilibre de la relation individu / puissance publique se solde toujours au profit du premier, la notion d'intérêt général remplissant ici un rôle justificatif. Ensuite parce qu'il faut rappeler que l'histoire contemporaine du droit public semble aller dans le sens d'une meilleure prise en compte des intérêts des individus. Sans être exclusive de tout autre type de considérations, l'influence des droits fondamentaux via les mouvements de constitutionnalisation et d'européanisation des branches du droit, notamment donc du droit public, s'avère déterminante.

Le droit public n'est donc pas étranger au phénomène global de promotion de la vulnérabilité en droit français. Mais c'est d'une vulnérabilité innommée dont il est question ici, puisque la prise en compte de la notion s'opère sans que le mot ou le langage de la vulnérabilité ne soient explicitement employés. Cette prise en compte de la vulnérabilité innommée constitue en quelque sorte le pré carré du droit public, puisqu'elle n'est ni plus ni moins que la conséquence du rapport de déséquilibre et de soumission qui constituent le cœur de la matière. Reste que le passage d'une vulnérabilité innommée à une vulnérabilité nommée - car liée à l'usage du vocabulaire dans les discours juridictionnels ou heuristiques du droit public - a de quoi interroger. Une chose en effet est de dire que le droit public repose sur la vulnérabilité, une toute autre est de définir ce concept, autrement dit de savoir ce qui se cache derrière la chose désormais signifiée. L'usage de l'expression transforme ainsi la relation qui s'établit entre les deux objets. S'installe dès lors par une sorte d'effet de miroir, une interrogation mutuelle du droit public et de la vulnérabilité. De telle sorte que si le droit public interroge la notion de vulnérabilité (I), celle-ci en retour en vient à questionner le droit public tant dans ses concepts que dans la protection que ce dernier est susceptible de lui offrir (II).

\section{La vulnérabilité interrogée par le droit public}

Le droit public, en tant que droit de la puissance publique, ne peut ignorer l'idée de vulnérabilité. Droit inégalitaire s'il en est, le droit public est implicitement fondé sur la notion de vulnérabilité des gouvernés face au pouvoir (A). Ce qui ne veut pas dire pour autant que l'émergence du vocabulaire de la vulnérabilité au cours des dernières années n'engendre pas certains problèmes notamment dans la délimitation et la définition de ce nouveau concept (B).

\section{A. La prise en compte implicite de la vulnérabilité}

S’il est vrai que la notion de vulnérabilité a fait une entrée remarquée en droit privé, on ne saurait cependant oublier que cette notion se situe également au cœur du droit public. Encore faut-il souligner que, pendant longtemps, c'est d'une vulnérabilité innomée dont il a été question. Loin d'être un détail dans l'édification du droit public, la prise en compte de la vulnérabilité constitue au contraire l'une des pierres angulaires de réflexion dans la construction de la théorie moderne de l'État et du statut de l'individu face au pouvoir. Dès le XVI ${ }^{\mathrm{e}}$ siècle les théories contractualistes cherchent à concilier l'abandon de l'état de nature au profit d'un état social et l'exercice légitime du pouvoir au sein de la structure politique. Hobbes est sans doute celui qui a le mieux souligné la fragilité de l'être humain tant dans l'état de nature que dans l'état social où prédomine un pouvoir tentaculaire et oppresseur auquel l'auteur prête les traits du Léviathan ${ }^{9}$. On peut sans doute fortement critiquer le caractère anxiogène et pessimiste de la philosophie hobbesienne, mais nul autre que lui n'a le mieux saisi le pouvoir de domination de l'État sur les individus. La philosophie des Lumières, à laquelle participent Locke et Rousseau, mais aussi les révolutions du XVIII ${ }^{\mathrm{e}}$ siècle au premier rang desquelles figure celle de 1789 et sa Déclaration des droits de l'homme et du citoyen, aboutiront à réévaluer les rapports entre gouvernés et gouvernants en assignant au pouvoir politique une contrainte de but: la préservation des libertés. Certes, l'État dispose toujours du monopole de la violence légitime, selon les mots du sociologue allemand Max Weber, mais il est désormais sommé, au moins théoriquement, de respecter les droits que les individus détiennent naturellement ${ }^{10}$. La naissance de l'État de droit au début du XX $\mathrm{XX}^{\mathrm{e}}$ siècle renforcera encore un peu plus la limitation du pouvoir de l'État. C'est ainsi que Léon Duguit analyse lui aussi l'État comme une structure de domination ${ }^{11}$,

7. Dictionnaire des droits de l’homme, J. Andriantsimbazovina, H. Gaudin, J.-P. Marguénaud, S. Rials, F. Sudre (dir.), Paris, Presses universitaires de France (Quadrige), 2008.

8. TC, 8 février 1873 , Blanco, Recueil Sirey, 1873, II, p. 153.

9. T. Hobbes, Léviathan, G. Mairet (trad.), Paris, Gallimard (Folio. Essais), 2009.

10. Sur ce point, voir N. Bobbio, Libéralisme et démocratie, Paris, Cerf (Humanités), 1996, p. 15-21, où l'auteur fait le lien entre les théories contractualistes et la naissance des droits de l'homme.

11. L. Duguit, Traité de droit constitutionnel, $3^{\mathrm{e}}$ éd., Paris, Fontemoing, 1927, t. I, \$63, p. 671: «Que la puissance gouvernante appartienne à un individu, à une classe, à la majorité numérique des membres du corps social, à des groupements secondaires, elle est une puissance de fait, pas autre chose». 
[...] un processus de différenciation entre gouvernants et gouvernés [,] [l]es premiers se trouvant détenteurs d'une force leur permettant d'occuper cette position ascendante et surplombante face à ceux auxquels ils peuvent ainsi imposer leur volonté ${ }^{12}$.

La «puissance de contrainte» tient une place majeure dans sa pensée. La «plus grande» force est pour lui «l'élément essentiel de tout État ${ }^{13}$, considérant que «les gouvernants ont toujours été, sont et seront toujours les plus forts en fait ${ }^{14}$. Face à la force se trouve nécessairement la faiblesse, la fragilité et par voie de conséquence une certaine idée de la vulnérabilité des gouvernés conduisant à analyser la Constitution d'un État comme la domination des gouvernants sur les gouvernés, donc des plus forts sur les plus vulnérables. Sur ce point, il est intéressant de constater que Duguit réfute le principe d'une autolimitation de l'État. Selon le père de la théorie du service public, en effet, l'autolimitation de l'État n'est qu'une fiction qui consiste à cacher le fait que «l'exercice du pouvoir implique toujours une relation du supérieur à l'inférieur ${ }^{15}$.

Toutefois, même au sein de la théorie de l'État de droit, l'idée de vulnérabilité, sans non plus là encore être nommée, est décelable. Certes, l'État de droit peut se définir comme un système institutionnel dans lequel la puissance publique est soumise au droit. Un tel système suppose, d'ailleurs, l'égalité des sujets devant les normes juridiques et l'existence de juridictions indépendantes. Mais précisément, la domination des uns sur la vulnérabilité des autres s'en trouve ainsi atténuée par la volonté d'établir l'égalité de tous devant la loi. Les normes juridiques sont hiérarchisées de telle sorte que la puissance de l'État s'en trouve limitée. Dès lors, la vulnérabilité n'a pas disparu de la conception de l'État puisqu'il s'agit justement de limiter la domination des gouvernants sur les gouvernés. La notion de vulnérabilité est d'autant plus présente que l'État de droit ne saurait s'envisager sans la reconnaissance de droits au bénéfice des personnes. Or, reconnaître ces droits implique d'identifier en amont des situations de faiblesse, d'identifier des vulnérabilités à protéger. La vulnérabilité est en effet intrinsèque à l'émergence des droits fondamentaux, là encore domaine majeur du droit public. La reconnaissance de ces droits, qui a jalonné le $\mathrm{XX}^{\mathrm{e}}$ siècle, a d'ailleurs contribué à faire évoluer le rôle de l'État vers un État providence impliquant qu'il agisse pour prendre en charge la (les) vulnérabilité(s) identifiée(s). Le droit constitutionnel s'avère bien sûr le premier réceptacle de ces transformations idéologiques et politiques quant à la conception du pouvoir, notamment en se proposant non seulement de fixer le statut des gouvernants, mais aussi les droits et libertés auxquels peuvent prétendre les gouvernés ${ }^{16}$. La montée en puissance de la justice constitutionnelle en France au cours des années $1970^{17} \mathrm{a}$, dans une large mesure, permis la mise en effectivité des catalogues constitutionnels des droits et libertés et ce, tant dans le cadre du contrôle a priori qu'a posteriori $i^{18}$ de constitutionnalité des lois.

Le droit administratif offre lui aussi des exemples d'une prise en compte de la vulnérabilité innommée. En effet, le droit administratif met en scène la relation déséquilibrée qui s'opère entre l'administration d'une part et les administrés d'autre part; relation par conséquent profondément inégalitaire. C'est d'ailleurs la raison d'être du droit administratif; si l'administration doit être soumise à des règles spéciales - le droit administratif -, c'est parce qu'elle est dans une situation très différente de celle des particuliers: se manifestant comme puissance publique, elle peut imposer unilatéralement des droits et des obligations aux administrés. Il existe un rapport d'autorité entre l'administration et les administrés, la première pouvant commander aux seconds. Dans la continuité des développements précédents la supériorité de l'administration vis-à-vis des administrés induit la faiblesse, et par voie de conséquence une certaine vulnérabilité de ces derniers, même si sa légitimité tient dans la poursuite de l'intérêt général. Les outils au service de l'action de l'administration en attestent. Outre le procédé autoritaire de l'acte administratif unilatéral qui s'impose aux administrés indépendamment de leur consentement, le contrat administratif, bien que plus consensuel, avance à visage masqué. En effet, les obligations qui en découlent pour le/les cocontractant(s) de l'administration ne trouvent pas leurs sources dans la seule et commune intention des parties. La volonté unilatérale de l'administration trouve là aussi largement à s'appliquer. Le contrat administratif est en effet lui-même, au nom de l'intérêt général, inégalitaire, ce qui le dissocie profondément du contrat de droit privé qui repose sur l'autonomie et l'égalité des parties (du moins en théorie). Le cocontractant de l'administration se retrouve ainsi dans une position de faiblesse, en conséquence de vulnérabilité. Dans le cadre du contrat administratif, l'administration dispose de pouvoirs importants, parmi lesquels on compte un pouvoir de direction, de contrôle, de sanction ou même encore de modification unilatérale. C'est précisément la raison pour laquelle,

12. E. Travers, «Volonté et puissance étatiques. Duguit critique de Rousseau, Kant et Hegel», Revue de la recherche juridique, $\mathrm{n}^{\circ} 3,2004, \mathrm{p} .1711$.

13. L. Duguit, Traité de droit constitutionnel, $\$ 1$, p. 3 : «On est ramené à l'élément essentiel de tout État: la plus grande force. Elle peut être matérielle ou morale; mais même lorsqu'elle n'est que morale, elle se traduit toujours par une puissance de contrainte. Il n'y a différenciation entre gouvernants et gouvernés que lorsque les gouvernants peuvent en fait, imposer par la contrainte leur volonté aux gouvernés».

14. Ibid., $\$ 62$, p. 656 .

15. E. Travers, «Volonté et puissance étatiques...», p. 1718.

16. Qu'il nous soit possible ici de renvoyer simplement à l'article 16 de la Déclaration des droits de l'homme et du citoyen du 26 août 1789 : «Toute société dans laquelle la garantie des droits n'est pas assurée, ni la séparation des pouvoirs déterminée, n'a point de Constitution».

17. Voir CC, déc. $\mathrm{n}^{\circ}$ 71-44 DC du 16 juillet 1971, Liberté d'association.

18. Nous faisons ici référence aux articles 61 et $61-1$ de la Constitution. 
à défaut d'égalité, le juge administratif a construit des mécanismes permettant à tout le moins d'assurer une certaine équité dans ces rapports contractuels. Face à de telles prérogatives de l'administration, il est apparu indispensable de faire bénéficier son cocontractant d'un droit à un certain "équilibre financier ${ }^{19}$, lui permettant, dans des hypothèses bien encadrées, de percevoir une indemnisation en vertu, par exemple, de la théorie de l'équation financière ${ }^{20}$, du fait du prince ${ }^{21}$ ou encore de l'imprévision ${ }^{22}$.

Le développement exponentiel de la responsabilité administrative et la "complexification des solutions ${ }^{23}$ tournés vers une meilleure indemnisation des victimes de l'action des autorités administratives révèlent également la prise en compte d'une vulnérabilité qui n'est pas nommée en tant que telle mais qui est sous-entendue dans le statut de victime. Le juge administratif déploie de nombreux mécanismes pour pallier cet état, notamment dans le cadre de la responsabilité sans faute, ayant fait émerger un "phénomène de socialisation des risques » afin de

[...] supporter la charge d'une indemnisation des préjudices qui excèdent, tant par leur particularité que par leur degré de gravité, ce que chacun doit supporter dans l'intérêt général ${ }^{24}$.

La responsabilité pour risque porte ainsi en elle la notion de vulnérabilité des administrés dont il convient de tenir compte pour mieux les indemniser. Cette vulnérabilité n'est d'ailleurs pas nécessairement attachée à la situation dans laquelle se trouve la personne, «le risque danger» se révélant "par l'activité d'une personne ou l'usage qui est fait d'une chose ${ }^{25}$. Par ailleurs, et de façon plus générale, certains auteurs, comme Maryse Deguergue, relèvent que

Le renforcement de l'effectivité des droits par l'engagement de la responsabilité administrative [...] bénéficie prioritairement à certaines catégories de citoyens en position de vulnérabilitée ${ }^{26}$,

ce qui fera l'objet d'une étude plus approfondie dans les développements qui vont suivre ${ }^{27}$.
On pourrait multiplier les exemples d'une vulnérabilité innommée dans les diverses branches du droit public. Il n'en reste pas moins que la vulnérabilité demeure difficile à cerner en tant que notion, cela d'autant plus que le droit a beaucoup évolué ces dernières années dans sa façon de l'appréhender et la saisir.

\section{B. Les contours incertains de la notion de vulnérabilité}

La prise en compte d'un certain état de fragilité des personnes en droit public n'empêche pas toutefois de s'interroger sur les contours de la notion de vulnérabilité telle qu'elle apparaît dans les discours relatifs à cette branche du droit. À dire vrai, la chose paraît à ce jour d'autant plus nécessaire que le vocabulaire de la vulnérabilité se trouve de plus en plus utilisé dans la langue publiciste, et ce quel que soit le type de support utilisé. En effet, ni le jurislateur, ni la doctrine ne semblent aujourd'hui ignorer la terminologie de la vulnérabilité, sans que l'on sache exactement cependant où placer le curseur de ce qui constitue l'état ou la situation de vulnérabilité. C'est donc, comme souvent, l'emploi du mot ou plus largement du vocabulaire lié au champ lexical de ce syntagme qui fait que l'on en soit venu à interroger l'étendue de la vulnérabilité. Or, la détermination de ce que recouvre juridiquement cette expression s'avère mal aisée. Cela pour plusieurs raisons. En premier lieu, il faut observer que l'étymologie des mots «vulnérables» ou «vulnérabilités» ne donnent que fort peu d'éléments quant aux contours de l'objet étudié. Pour l'essentiel en effet, il s'agira pour les auteurs de rappeler que la vulnérabilité est une fragilité. Cette acception classique, qui est celle reçue par la majorité des membres de la doctrine juridique comme cadre sémantique à leur réflexion ${ }^{28}$, pose d'emblée un problème dès lors que toute personne se trouve par définition en situation de vulnérabilité ou, pour le dire d'une manière inversée, nul n'est invulnérable. En d'autres termes, rechercher les contours de la vulnérabilité au sens juridique du terme implique de dépasser ce premier écueil pour que soient

19. CE, 27 octobre 2010, Syndicat intercommunal des transports publics de Cannes, L'actualité juridique. Droit administratif, 2010 , p. 2076.

20. Il s'agit de l'hypothèse dans laquelle l'administration a utilisé son pouvoir de modification unilatérale du contrat pour augmenter les obligations de son cocontractant (CE, 21 mars 1910, Compagnie générale française des tramways, Recueil Lebon, p. 216, concl. L. Blum).

21. Il s'agit cette fois de l'hypothèse dans laquelle l'administration aura modifié unilatéralement et indirectement les conditions d'exécution du contrat (CE, 29 décembre 1905, Bardy, Recueil Lebon, p. 1014, concl. J. Romieu).

22. Cette hypothèse est celle dans laquelle se réalise un aléa anormal dû à la survenance d'un événement imprévisible et difficilement résistible (CE, 30 mars 1916, Compagnie générale d'éclairage de Bordeaux, $\mathrm{n}^{\circ} 59928$ ).

23. P. Gonod, «À propos de la responsabilité administrative», Mouvements, n² 29, 2003, p. 35.

24. Ibid. L'auteure poursuit en indiquant que la mise en place par le législateur de fonds de solidarité visant à réparer les dommages subis collectivement a d'ailleurs répondu à cette forte tendance laissant envisager qu' «une logique de solidarité [...] par suite se substitu[ait] à une logique de responsabilité».

25. M. Deguergue, «Regard sur les transformations de la responsabilité administrative», Revue française d'administration publique, ${ }^{\circ}$ 147, 2013, p. 579. L'auteure fait référence aux hypothèses de responsabilité administrative sans faute au sein des hôpitaux publics: CE, Ass., 9 avril 1993, Bianchi, Revue française de droit administratif, 1993, p. 573, concl. S. Daël; CE, 9 juillet 2003, APHP c. Marzouk, Recueil Lebon, p. 338 ; L'actualité juridique. Droit administratif, 2003, p. 1946, note M. Deguergue.

26. M. Deguergue, «Regard sur les transformations...», p. 583.

27. II.B ci-dessous.

28. Pour des exemples, voir F. Fiechter-Boulvard, «La notion de vulnérabilité et sa consécration par le droit », in Vulnérabilité et droit: le développement de la vulnérabilité et ses enjeux en droit, F. Cohet-Cordey (dir.), Grenoble, Presses universitaires de Grenoble, 200o, p. 13-32; F.-X. Roux-Demare, "La notion de vulnérabilité...". 
recherchées, au-delà de la fragilité qui nous affecte tous, des causes spécifiques de vulnérabilité. Or, sur ce point également, les choses achoppent. C'est ainsi que les auteurs se rassemblent autour de deux fondements au moins de vulnérabilité. En premier lieu, la vulnérabilité est présentée comme une qualité personnelle ${ }^{29}$. Seraient dès lors «vulnérables» les personnes handicapées, celles souffrant de troubles mentaux, les personnes âgées ( $a$ fortiori lorsque ces dernières sont en situation de dépendance), les enfants, etc. Cette première catégorisation semble à première vue relativement homogène. Elle suscite en réalité un certain nombre d'interrogations pour qui accepte de l'envisager pour ce qu'elle est: une suite ininterrompue de "fragilités » et de maux hétéroclites. Il est en effet délicat de placer dans une même catégorie des personnes dont l'état de vulnérabilité semble perpétuel (handicap, troubles mentaux, personnes âgées) et des personnes qui, à l'instar des mineurs, relèvent d'un état de vulnérabilité que l'on serait tenté de qualifier de transitoire ou de passager. En second lieu, il est intéressant de constater que, parallèlement à cette première approche, un second sens de la vulnérabilité tend à se développer qui envisage cette fois-ci la notion dans un sens contextuel et non plus personnel. Pour le dire autrement, la vulnérabilité serait le résultat tantôt d'un risque pesant sur la personne, tantôt d'un rapport de force défavorable à cette personne. Cette extension du champ de la vulnérabilité à des sujets qui, en tant que tels, ne connaissent pas de fragilité personnelle, impose une dilution des contours de la notion. D'abord parce que définie de la sorte la vulnérabilité entretient des rapports étroits avec le principe de non-discrimination avec lequel pourtant elle ne se confond pas ${ }^{30}$. Ensuite parce que pensée en des termes contextuels la notion de vulnérabilité est de nature à se montrer accueillante. La vulnérabilité dont il est question ici peut ainsi tout aussi bien toucher des personnes physiques que des personnes morales. Personnes morales de droit privé, mais aussi, potentiellement, de droit public. Aussi bien, l'État pourrait parfaitement être placé en situation de vulnérabilité selon cette approche. L'hypothèse n'est pas à exclure pour deux raisons. D'abord parce que l'État peut dans certains cas être placé dans la même situation que celle de particuliers (ainsi de l'État gestionnaire de son domaine privé). Ensuite, et surtout, parce qu'il est désormais acquis que des puissances concurrentes se développent face à l'État, à l'instar de certaines personnes privées telles que les GAFAM ${ }^{31}$, dont la puissance (financière mais aussi et surtout technologique) peut constituer une menace, voire un risque pour la puissance de l'État.
En outre, un autre écueil doit être pris en compte dans la construction d'un concept juridique de vulnérabilité : celui de son caractère ambivalent. En effet, la vulnérabilité peut parfois servir de support à l'édiction de mesures particulières favorables à la personne dont l'état de vulnérabilité a été reconnu. Par exemple, dans une affaire D. H. et autres c. République tchèque, la grande chambre de la Cour européenne des droits de l'homme affirme que les Roms sont «[...] une minorité défavorisée et vulnérable» avant de souligner que, pour cette raison, le peuple rom a «dès lors besoin d'une protection spéciale $»^{32}$. Mais dans certains cas l'état de vulnérabilité d'une personne peut aussi donner lieu à des mesures négatives, prises, donc, à l'encontre de cette personne. C'est le cas lorsque l'individu concerné exerce des fonctions éminentes. En droit constitutionnel, l'altération des fonctions cognitives et intellectuelles du chef de l'État est analysée comme étant un cas de vacance ou d'empêchement définitif donnant lieu à l'organisation d'élections présidentielles ${ }^{33}$. L'objectif de ces mesures est bien sûr d'assurer la continuité de l'État ainsi que le bon exercice du pouvoir au sein de l'exécutif. Plus récemment, et dans un autre ordre d'idée, le mot «vulnérabilité» a été employé dans son acception négative dans une ordonnance de référé rendue par le tribunal administratif de Paris ${ }^{34}$. En cause devant le juge, les mesures restrictives prises à l'encontre d'un officier de police suspecté de radicalisation. Rappelant l'attentat survenu à la préfecture de police quelques semaines plus tôt, l'écho médiatique que cette attaque avait suscité et l'émotion qu'elle avait provoquée, le juge considère que le préfet de police était fondé à prendre des mesures conservatoires envers «ceux des agents qui présentaient des indices de vulnérabilité, même faibles, en matière de radicalisation qui seraient de nature à faire naître des craintes chez leurs collègues de travail». On le voit, la notion de vulnérabilité est ici employée dans un sens péjoratif. La référence à la vulnérabilité est donc de nature à engendrer des attitudes différentes de la part des pouvoirs publics selon les cas. Faut-il d'ailleurs s'en étonner? Nous ne le pensons pas. Le Conseil d'État avait déjà eu l'occasion de mettre implicitement cette idée en lumière dans la jurisprudence Commune de Morsang-surOrge dans laquelle, prenant acte du handicap physique du requérant, la haute juridiction administrative avait souligné l'atteinte au principe de dignité de la personne humaine. En l'espèce c'est bien la prise en compte de ce handicap qui conduit à l'interdiction du spectacle de lancer de nain ${ }^{35}$.

29. L'utilisation de l'image d'Achille dans l'article de Frédérique Fiechter-Boulvard est de ce point de vue tout à fait explicite de ce que la vulnérabilité s'attache avant tout à la personne; voir, ainsi, F. Fiechter-Boulvard, «La notion de vulnérabilité... », p. 13.

30. Sur ce point, voir l'article 225-1 du Code pénal et la liste des critères utilisés par cette disposition.

31. Il s'agit d'un acronyme afin de désigner les sociétés que sont Google, Amazon, Facebook, Apple et Microsoft.

32. Cour EDH, GC, 13 novembre 2007, D. H. et autres c. République tchèque, $\mathrm{n}^{\circ}$ 57325/00, $\$ 182$.

33. Article 7 de la Constitution du 4 octobre 1958

34. TA Paris, ord., 3 janvier $2020, \mathrm{n}^{\circ} 1926536 / 5$.

35. CE, Ass., 27 octobre 1995, Commune de Morsang-sur-Orge, n 136727 : "Considérant que l'attraction de "lancer de nain" consistant à faire lancer un nain par des spectateurs conduit à utiliser comme un projectile une personne affectée d'un handicap physique et présentée comme telle; que, par son objet même, une telle attraction porte atteinte à la dignité de la personne humaine». 
Ainsi, si la vulnérabilité est indéniablement au cœur du droit public, celui-ci l'implique et la saisit de façon très hétérogène. Mais en la faisant émerger de plus en plus explicitement, c'est le droit public lui-même qui évolue sous le coup de la vulnérabilité.

\section{Le droit public interrogé par la vulnérabilité}

La prise en compte de la vulnérabilité interroge en retour le droit public. Dans ses concepts tout d'abord dès lors que la prise en compte de la vulnérabilité impose certains infléchissements des principes qui sont au cœur du droit public (A). Mais aussi interrogation sur le rôle des garanties juridictionnelles dès lors que le juge est parfois saisi de l'appréciation de cette vulnérabilité et des implications réelles ou supposées de celle-ci sur le travail juridictionnel (B).

\section{A. La redéfinition des concepts fondateurs}

La notion de vulnérabilité, bien qu'aux contours incertains, impose une transformation, un changement de regard sur certains concepts centraux du droit public.

Il en va ainsi notamment du principe d'égalité. L'égalité formelle, ou abstraite, qui est l'égalité juridique conformément au principe selon lequel les mêmes lois s'appliquent à tous ${ }^{36}$, a longtemps prévalu au détriment d'une analyse pragmatique des situations visant à une égalité réelle vérifiable. L'égalité de droit a pour effet de masquer les inégalités de fait dans lesquelles des situations de vulnérabilité sont identifiables. Les jurisprudences administrative ${ }^{37}$ et constitutionnelle ${ }^{38}$ ont par la suite reconnu l'idée selon laquelle des situations différentes peuvent se voir appliquer des règles de droit différentes. La discrimination qui s'opère alors ici par la voie de la différenciation ${ }^{39}$ a pour objet de mieux appréhender les différences des usagers du service public, des agents, des entreprises partenaires, etc. La notion de vulnérabilité n'est a priori pas présente dans cette dimension (ou dérogation?) du principe d'égalité $4^{\circ}$. Mais l'heure ne semble plus seulement être à celle de savoir si le droit opère des distinctions. La question est davantage aujourd'hui celle de savoir si ces distinctions produisent bien pour effet réel une meilleure égalité ${ }^{41}$. Une plus grande prise en compte de la vulnérabilité des personnes conduit à faire évoluer le principe d'égalité lequel tend de plus en plus à être doublé d'un principe de non-discrimination. Ce principe de non-discrimination, qui désigne l'interdiction de traiter moins favorablement une personne en raison de critères, réels ou supposés, tels que l'apparence, la croyance, l'âge ou le sexe, met davantage en relief la notion de vulnérabilité puisqu'il s'agit d'interdire de traiter défavorablement des personnes vulnérables. La vulnérabilité transforme ainsi progressivement le principe d'égalité.

La prise en compte contemporaine par le droit des inégalités de fait pour assurer encore davantage l'égalité de droit conduit ainsi à une évolution majeure de la conception du principe d'égalité. Aujourd'hui l'enjeu est d'aller au-delà de la conception classique du principe pour mieux envisager les situations individuelles et prendre en compte la situation concrète de l'individu. Devant l'insuffisance du principe de l'égalité juridique, il est apparu nécessaire de redéfinir les droits pour envisager l'individu dans son environnement social et dans sa situation particulière, afin de lui assurer une plus grande protection. Cela a conduit à adopter une vision ultra-subjectiviste des droits consistant à reconnaître des droits spécifiques, sur mesure, au cas par cas. Yaël Attal-Galy exprime cela par la «nécessité d'adapter le droit au particularisme des situations individuelles ${ }^{42}$, le

[...] souci de prendre en compte les réalités et les situations concrètes de certaines catégories d'individus défavorisées souvent menacées par l'exclusion, les discriminations, la misère $[\ldots]^{43}$.

L'enjeu étant de parvenir à l'égalité réelle.

C'est ainsi que l'on assiste à une évolution qui consiste à passer de l'individualisme, qui envisage l'individu comme un sujet autonome mais abstrait, c'est-à-dire non situé dans un contexte social, au personnalisme qui considère la personne humaine en tant que travailleur, femme, membre d'un groupe particulier, ce qui se traduit par l'insertion de cet individu dans différents groupes (famille, communauté de travail par exemple). La personne est envisagée dans sa totalité: à la fois comme "individu» et comme "être social», ce dernier aspect conduisant à reconnaître des droits collectifs. Ce sont ainsi des vulnérabilités individuelles mais aussi collectives qui sont prises en compte.

La doctrine solidariste, la doctrine marxiste avaient déjà mis en exergue ces nécessités, et en permettant de saisir les réalités sociales, elles ont incité à l'émergence de nouveaux droits: les droits économiques, sociaux et culturels dont certains sont apparus avec la rédaction du

36. En vertu de l'article premier de la Déclaration des droits de l'homme et du citoyen de 1789: «Tous les êtres humains naissent libres et égaux en dignité et en droit».

37. CE, sect., 10 mai 1974, Denoyez et Chorques, $\mathrm{n}^{\circ} 88032$.

38. CC, déc. $\mathrm{n}^{\circ}$ 79-107 DC du 12 juillet 1979, Ponts à péages.

39. Voir en ce sens M. Borgetto, "Égalité, différenciation et discrimination: ce que dit le droit», Informations sociales, $\mathrm{n}^{\circ}$ 148, 2008 , p. 8-17.

40. B. Seiller, "Contribution à la résolution de quelques incohérences de la formulation prétorienne du principe d'égalité», in Le droit administratif: permanences et convergences. Mélanges en l'honneur de Jean-François Lachaume, Paris, Dalloz, 2007, p. 979.

41. Voir en ce sens, sur la question de l'égalité entre les hommes et les femmes, S. Hennette-Vauchez, D. Roman, Droits de l'homme et libertés fondamentales, $1^{\text {re }}$ éd., Paris, Dalloz, 2013, p. 625.

42. Y. Attal-Galy, Droits de l'homme et catégories d'individus, Paris, LGDJ (Thèses), 2004, p. 61.

43. Ibid. 
préambule de la Constitution de 1946. Ce texte est fondamental en ce qu'il précise l'étendue du principe d'égalité en désignant explicitement les catégories d'individus qui nécessitent, en raison de leur vulnérabilité, un traitement particulier: la personne handicapée, l'étranger, la femme, l'enfant, la personne malade... La formulation de l'alinéa 3 du texte est relativement évocatrice de ce que la conception égalitaire de 1789 ne suffit pas. En effet, il dispose que «la loi garantit à la femme, dans tous les domaines, des droits égaux à ceux de l'homme». Cette nouvelle disposition doit permettre au législateur d'accomplir l'égalité économique et sociale pour faire disparaître dans tous les domaines les dispositions discriminatoires à l'égard des femmes. Il en va de même pour les étrangers en vertu de l'alinéa 4 : il s'agit de protéger ceux qui sont persécutés dans leur pays et de leur reconnaître le droit d'asile. Plusieurs droits fondamentaux sont ainsi proclamés, comme le droit au travail. Le principe d'égalité dans l'emploi est intéressant en ce qu'il est décliné sous la forme du principe de nondiscrimination en raison des origines, des opinions ou des croyances des travailleurs. Sont par ailleurs reconnus la liberté syndicale et le droit de grève. Les individus sans emploi ou vivant dans la précarité font également l'objet d'une attention particulière. La prise en compte de la vulnérabilité dans l'énoncé du droit, des droits, conduit nécessairement à porter sur eux un regard nouveau, à faire émerger de nouvelles conceptions, l'objectif recherché étant l'amélioration des conditions de vie de ces personnes en situation de vulnérabilité. Comme l'écrit Attal-Galy, «la catégorisation du droit permet aux plus défavorisés de bénéficier d'un traitement plus adapté au particularisme de leur situation ${ }^{44}$. Cette approche des droits catégoriels est aussi celle des textes internationaux. En témoignent, par exemple, la Convention sur l'élimination de toutes les formes de discrimination à l'égard des femmes (1981) ou la Convention relative aux droits de l'enfant (1989). L'insuffisante protection des textes à portée générale fondés sur une conception universelle de la personne humaine a conduit là aussi à la particularisation des droits au niveau international.

Les décisions du juge administratif sont empreintes d'une telle évolution du droit et des droits. Pour ne prendre qu'un exemple, en matière de handicap, le juge procède à une analyse très particulière de la situation. Dans une affaire, il a en effet mis en exergue la nécessité de prendre en compte la situation spécifique de handicap en exigeant, pour fixer le taux de la prime individuelle d'un magistrat qui a la qualité de travailleur handicapé, que l'administration tienne compte de son handicap non seulement pour déterminer le volume et la nature des tâches qui lui sont assignées mais aussi pour apprécier, au vu des objectifs ainsi définis par rapport à ses capacités, la contribution de l'intéressé au bon fonctionnement de l'institution judiciaire ${ }^{45}$. Le handicap fait aussi l'objet d'une prise en compte particulière en cas d'autorisation administrative de licencier un salarié protégé ${ }^{46}$. De même, dans une procédure de référé-suspension, le tribunal administratif a pris en compte la situation de handicap pour caractériser à la fois l'urgence et le doute sérieux quant à la légalité de la décision:

[...] eu égard à la situation de vulnérabilité qui est la sienne du fait de son handicap, le requérant doit être regardé comme justifiant d'une situation d'urgence [...]. L'affectation de M. Bore a été opérée sans examen particulier de sa situation de handicap et est de nature à faire naitre un doute sérieux quant à la légalité de la décision attaquée ${ }^{47}$.

On assiste ainsi indubitablement à une approche plus empirique et casuistique des droits pour mieux prendre en compte la / les vulnérabilité(s), l'objectif étant d'assurer une plus grande protection de la personne, de l'individu.

Outre une redéfinition des concepts fondateurs du droit public, la notion de vulnérabilité interroge également le rôle des garanties juridictionnelles dès lors que le juge administratif est saisi d'affaires dans lesquelles il est amené à apprécier des situations de vulnérabilité et à en tirer des conséquences pratiques.

\section{B. Le rôle des garanties juridictionnelles}

L'émergence contemporaine de la notion de vulnérabilité en droit public se solde par une certaine forme d'adaptation des garanties offertes par le droit public aux personnes vulnérables. Certes, cette prise en compte de la vulnérabilité est encore balbutiante et pour tout dire imparfaite. Ainsi la vulnérabilité d'une personne n'est-elle pas toujours de nature à produire des effets protecteurs vis-à-vis des individus concernés ${ }^{48}$. Mais il apparaît aussi que la vulnérabilité d'un requérant soit positivement prise en compte. Cette prise en compte peut résulter soit de l'appréciation des conditions de recevabilité d'une action, soit encore de la solution proposée par le juge saisi de la demande. La pratique du référé-liberté est topique du premier cas de figure via notamment les conditions d'urgence et de gravité de l'atteinte exposées au sein de l'article L. 521-2 du Code de justice administrative. Le juge administratif se livre en effet à une appréciation in concreto de chacun de ces deux critères. Cela ne signifie toutefois pas l'abdication de la part du juge administratif de toute forme de réalisme, a fortiori lorsque la mesure portée devant lui nécessite l'intervention active des pouvoirs publics. Ainsi, dans son ordonnance Karamoko A., le Conseil d'État considère que la carence de l'État dans la mise en œuvre du droit à un hébergement d'urgence est de

44. Ibid., p. 65

45. CE, 11 juillet 2012, Vaulot Pfister, $\mathrm{n}^{\circ} 347703$.

46. CE, 3 juillet 2013, M. B., nº 349496; CAA Versailles, 23 février 2012, André, no 10 VEo3075.

47. TA Nantes, ord., 16 octobre 2014, Bore, no 1408215 .

48. CAA Nantes, $2^{\mathrm{e}}$ ch., 14 décembre $2018, \mathrm{n}^{\circ} 17 \mathrm{NT} 03961$. 
nature à faire naître une atteinte grave et manifestement illégale à une liberté fondamentale lorsque cette carence a des conséquences graves pour la personne intéressée. Mais il retient également dans la foulée qu'il

[...] incombe au juge des référés d'apprécier dans chaque cas les diligences accomplies par l'administration en tenant compte des moyens dont elle dispose ainsi que de l'âge, de l'état de santé et de la situation familiale de la personne intéressée ${ }^{49}$.

C’est en appréciant ces éléments que le juge administratif prend en compte la vulnérabilité du requérant. S'agissant des détenus, le juge des référés a eu l'occasion à de multiples reprises de souligner leur vulnérabilité particulière $^{50}$. Toutefois cette reconnaissance ne va pas jusqu'à établir une présomption d'urgence dans le contentieux du référé-liberté dans les cas de placement à l'isolement ${ }^{51}$, alors pourtant que cette présomption existe dans le contentieux du référé-suspension ${ }^{52}$. Pour ce qui est de la prise en compte de la vulnérabilité du requérant dans la solution juridictionnelle, la jurisprudence de la Cour européenne des droits de l'homme s'avère d'une aide précieuse. Soucieuse en effet de procéder à une lecture dynamique de la convention et de ne point protéger des droits théoriques et illusoires mais au contraire "concrets et effectifs ${ }^{53}$, la Cour n'hésite pas le cas échéant à adapter ses standards de protection. Ainsi, dans l'arrêt Vincent c. France, la Cour reconnaît une violation de l'article 3 de la Convention européenne des droits de l'homme à l'égard d'un détenu paraplégique dans l'incapacité de circuler sans aide dans les locaux de la prison de Fresnes. Le juge de Strasbourg retient ici de manière tout à fait intéressante que cette violation du principe de prohibition des traitements inhumains et dégradants existe même si «rien ne prouve l'existence d'une intention d'humilier ou de rabaisser le requérant ${ }^{54}$. Dans l'affaire Jasinskis c. Lettonie, la Cour constate cette fois une violation de l'article 2 de la Convention après le décès d'une personne sourde et muette en cellule de dégrisement, le juge soulignant ici que, s'agissant d'une personne handicapée, la nécessité de garantir l'adéquation des conditions de détention à sa situation particulière s'avérait d'autant plus grande ${ }^{55}$. La Cour européenne des droits de l'homme est donc particulièrement attentive aux situations particulières et aux sujétions individuelles, comme le souligne par ailleurs Céline Ruet ${ }^{56}$.

Au terme de cet article, il ne fait pas de doute que la notion de vulnérabilité innerve le droit public, soit qu'elle en soit au fondement, soit qu'elle participe à sa transformation par la prise en compte nécessaire des considérations contemporaines qui visent à assurer une plus grande protection des personnes. Reste que ses contours sont sources d'interrogations tant elle est à la merci des pouvoirs publics qui la mobilisent et la manient selon les objectifs, divers, à atteindre. Notion polymorphe, qui peut se décliner au pluriel, la vulnérabilité

[...] est devenue une catégorie dominante d'expression des difficultés à être en société [...] en même temps qu'une catégorie agissante au nom de laquelle se déploie aujourd'hui une grande diversité de registres de l'action publique ${ }^{57}$.

Il n'en fallait pas moins pour identifier toute son équivocité et le champ d'étude qu'elle offre à la réflexion.

49. CE, ord., 10 février 2012, Karamoko A., nº 356456

50. Ainsi, CE, ord., 22 décembre 2012, Section française de l'observatoire international des prisons et autres, $\mathrm{n}^{\circ} 364584,364620,364621,364647:$ « [... ] qu'eu égard à la vulnérabilité des détenus et à leur situation d'entière dépendance vis-à-vis de l'administration, il appartient à celle-ci, et notamment aux directeurs des établissements pénitentiaires, en leur qualité de chefs de service, de prendre les mesures propres à protéger leur vie ainsi qu’à leur éviter tout traitement inhumain ou dégradant afin de garantir le respect effectif des exigences découlant des principes rappelés notamment aux articles 2 et 3 de la convention européenne de sauvegarde des droits de l'homme et des libertés fondamentales [...]». Sur cette ordonnance, voir O. Le Bot, «Référé-liberté aux Baumettes: remède à l'inertie administrative et consécration d'une nouvelle liberté fondamentale», La semaine juridique, édition générale, $\mathrm{n}^{\circ}$ 4, 21 janvier 2013, p. 139 sq. Pour une reprise de l'affirmation concernant l'état de vulnérabilité des détenus, voir CE, ord., 30 juin $2015, n^{\circ} 392043,392044$.

51. CE, ord., 20 novembre 2019, n 435785; B. David, «Isolement: de l'urgence présumée en référé-suspension mais non en référé-liberté», Actualité juridique. Pénal, $\mathrm{n}^{\circ} 1,2020$, p. $40 \mathrm{sq}$.

52. CE, ord., 7 juin 2019, $\mathrm{n}^{\circ} 426772$ : «Eu égard à son objet et à ses effets sur les conditions de détention, la décision plaçant d'office à l'isolement une personne détenue ainsi que les décisions prolongeant éventuellement un tel placement, prises sur le fondement de l'article 726-1 du Code de procédure pénale, portent en principe, sauf à ce que l'administration pénitentiaire fasse valoir des circonstances particulières, une atteinte grave et immédiate à la situation de la personne détenue, de nature à créer une situation d'urgence justifiant que le juge administratif des référés, saisi sur le fondement de l'article L. 521-1 du Code de justice administrative, puisse ordonner la suspension de leur exécution s'il estime remplie l'autre condition posée par cet article».

53. Cour EDH, 9 octobre 1979, Airey c. Irlande, $\mathrm{n}^{\circ} 6289 / 73, \S 24$.

54. Cour EDH, 24 octobre 2006, Vincent c. France, $\mathrm{n}^{\circ} 6253 / 03$.

55. Cour EDH, 21 décembre 2010, Jasinskis c. Lettonie, $\mathrm{n}^{\circ} 45744 / 08$.

56. C. Ruet, «La vulnérabilité dans la jurisprudence de la Cour européenne des droits de l'homme», Revue trimestrielle des droits de l'homme, 2015, p. 317 sq.

57. M.-H. Soulet, «Les raisons d'un succès. La vulnérabilité comme analyseur des problèmes sociaux contemporains", in Vulnérabilités sanitaires et sociales. De l'histoire à la sociologie, A. Brodiez-Dolino, I. von Bueltzingsloewen, B. Eyraud, C. Laval, B. Ravon (dir.), Rennes, Presses universitaires de Rennes, 2014, p. 59. 